EDITORIAL

\title{
EL USO DE UN PRODUCTO EN INVESTIGACIÓN FUERA DE UN ENSAYO CLÍNICO: EL CASO «VACUNAGATE»
}

\author{
USE OF EXPERIMENTAL VACCINE OUTSIDE OF CLINICAL \\ TRIAL: THE "VACUNAGATE" CASE \\ Percy Mayta-Tristán(101, Juan Pablo Aparco(i)2 \\ 1 Dirección General de Investigación, Desarrollo e Innovación, Universidad Científica del Sur, Lima, Perú. \\ 2 Centro Nacional de Alimentación y Nutrición, Instituto Nacional de Salud, Lima, Perú.
}

Citar como: Mayta-Tristán P, Aparco JP. El uso de un producto en investigación fuera de un ensayo clínico: el caso «Vacunagate». Rev Peru Med Exp Salud Publica. 2021;38(2):203-5. doi: https://doi. org/10.17843/rpmesp.2021.382.8694

Correspondencia: Percy MaytaTristán; Av. Brasil 2169 Dpto. 802, Jesús María, Lima; pmayta@cientifica.edu.pe

Recibido: $23 / 06 / 2021$ Aprobado: $30 / 06 / 2021$ En línea: 01/07/2021
En los últimos cien años, la pandemia por la COVID-19 ha sido la peor amenaza contra la salud pública, la economía y las actividades humanas en el mundo entero. El tener vacunas efectivas con prontitud es una necesidad, pero su desarrollo impone grandes desafíos médicos, científicos y éticos ${ }^{(1)}$. En dicho contexto, una vez superados los estudios de las fases preclínicas, los ensayos clínicos constituyen el estándar por excelencia para probar las vacunas experimentales contra la COVID-19.

En el Perú, se habían aprobado cuatro ensayos clínicos de fase 3 de vacunas contra la COVID-19, en uno de ellos se planificó reclutar a doce mil voluntarios para evaluar dos vacunas experimentales de virus inactivados, desarrolladas por la compañía china Sinopharm. Sin embargo, la ejecución del ensayo se vio comprometida en un escándalo político vinculado con la inoculación del producto en investigación a 470 personas por fuera del ensayo clínico, dicho suceso fue denominado «Vacunagate» (2).

La lista de vacunados incluía al presidente de la República y sus familiares; a altos funcionarios del Ministerio de Salud y la Cancillería relacionados con el proceso de compra y aprobación de las vacunas y con el manejo de la pandemia; a autoridades de las dos universidades donde se realizó el estudio (Universidad Peruana Cayetano Heredia y Universidad Nacional Mayor de San Marcos); al equipo de investigadores del ensayo, familiares, docentes e investigadores de ambas universidades que no eran parte del equipo de investigación e incluso a un representante de la Iglesia católica. Todos fueron vacunados antes de que la vacuna experimental estuviera aprobada y disponible como vacuna para su uso de emergencia en el Perú ${ }^{(3,4)}$.

Más allá de las implicancias legales ${ }^{(5)}$, según el informe de la Comisión Sectorial investigadora de la aplicación de la vacuna candidata contra la COVID-19 se cometieron potenciales infracciones al reglamento de ensayos clínicos y al código nacional de integridad científica ${ }^{(4)}$, así como a las normas éticas, deontológicas y legales establecidas en las instituciones de investigación implicadas. A cinco meses de hacerse público estos hechos, el propósito de este manuscrito es describir lo que se conoce sobre el uso de un producto en investigación fuera de un ensayo clínico en un contexto de pandemia.

En los inicios de la pandemia por la COVID-19 y antes del desarrollo, experimentación y uso de vacunas contra esta enfermedad, se analizó el uso de vacunas experimentales en el personal de investigación como una forma de protegerlos durante brotes epidémicos y contexto pandémico ${ }^{(6-8)}$. Se planteó la posibilidad de que el personal de salud, incluso los investigadores, accedan a estas vacunas experimentales, pero como parte del ensayo clínico, es decir, como voluntarios ${ }^{(8)}$. Esta propuesta se basa en la inclusión, como voluntarios de un ensayo clínico, del personal que conducía un estudio con una vacuna experimental contra el Ébola en Guinea en 2018. Esta fue una estrategia para incentivar el enrolamiento de participantes 
en el ensayo clínico ${ }^{(9)}$. Estas decisiones fueron planteadas como medidas excepcionales para situaciones particulares como las pandemias, pero en ningún caso se plantea el uso de la vacuna experimental en los investigadores, personal de salud y otras personas por fuera del ensayo clínico ${ }^{(8)}$.

El uso de intervenciones no probadas en la práctica clínica y usadas por fuera de un ensayo clínico se plantean en el artículo 37 de la Declaración de Helsinki ${ }^{(10)}$, y estaba regulado como uso compasivo en los artículos 115, 116 y 117 del Reglamento de Ensayos Clínicos (REC) del Perú de 2006. Para el uso compasivo, cada caso requería el consentimiento informado del paciente, un informe clínico del médico tratante que justifique la necesidad del tratamiento, la conformidad del director de la Institución donde se aplicaba el tratamiento y la autorización de la Dirección General de Medicamentos, Insumos y Drogas (DIGEMID) ${ }^{(11)}$. Las discusiones éticas vinculadas al uso compasivo de medicamentos fuera de ensayos clínicos están vigentes, más aún en situaciones excepcionales como una pandemia; sin embargo, esta forma de uso siempre debe estar enmarcado en la normativa de cada país ${ }^{(12,13)}$. La actualización del REC de 2017 retiró los artículos vinculados al uso compasivo de productos farmacéuticos o biológicos en el Perú (14).

$\mathrm{Al}$ inicio de la pandemia se analizó el uso compasivo de diferentes medicamentos para el tratamiento de la COVID-19, sobre todo ante la falta de evidencias sobre la conducta terapéutica para evitar muertes en pacientes con COVID-19 grave. Sin embargo, en estos análisis no se mencionan las vacunas como parte del uso compasivo en casos de COVID-19 ${ }^{(15,16)}$. Se discutieron las decisiones de los gobiernos de Rusia y China en aplicar sus vacunas experimentales a determinadas poblaciones como uso compasivo, aún sin tener los resultados de fase 3, y con base en los resultados de seguridad y capacidad inmunogénica de los ensayos de fase 1 y 2 , y bajo las regulaciones específicas de esos países ${ }^{\left({ }^{17}\right)}$. Asimismo, en Emiratos Árabes Unidos los primeros vacunados fueron autoridades o personas vinculadas al manejo de la pandemia, incluso antes de la autorización de su uso de emergencia, a manera de protección de quienes están más expuestos y como ejemplo para incentivar la vacunación, pero esta estrategia fue pública y transparente ${ }^{(18)}$.

En el caso del ensayo clínico de Sinopharm en el Perú, el protocolo de estudio incluyó 3200 dosis adicionales que no estaban destinadas a los voluntarios, sino a la protección del equipo de investigación y del personal relacionado al estudio, y solo cuando se tuviera evidencia de su eficacia y seguridad ${ }^{(4)}$. Esta estrategia de mitigación de riesgos para los investigadores no se ha verificado en ningún otro ensayo clínico con vacunas contra la COVID-19 en el Perú, aunque sí hay reportes de envíos de dosis adicionales por fuera de los ensayos clínicos realizados por otros laboratorios chinos, como Sinopharm, Sinovac y CanSino, en Latinoamérica ${ }^{(19)}$.

Por otro lado, no se justifica el envío de un producto en investigación fuera de las instalaciones del centro de investigación, situación prohibida por el REC ${ }^{(14)}$. No obstante, se transgredió esa disposición con el envío de 1200 dosis para la Embajada de China en el Perú. La comisión investigadora del Ministerio de Salud identificó que en todas las inspecciones que realizó el Instituto Nacional de Salud (INS) al ensayo clínico, el patrocinador no comunicó sobre el uso y destino de estas dosis adicionales y que tampoco estableció una regla de decisión sobre el acceso a este producto en investigación ${ }^{(4)}$.

Diferentes países del mundo ${ }^{(20,21)}$, incluyendo al Perú ${ }^{(22)}$, adoptaron medidas para agilizar los trámites regulatorios que permitan desarrollar ensayos clínicos en plazos más cortos. Sin embargo, el riesgo de acortar los plazos pudo generar que el Comité Nacional Transitorio de Ética en Investigación para la evaluación y supervisión ética de los Ensayos Clínicos de la enfermedad COVID-19, el INS y la DIGEMID no observaran algunos aspectos críticos en la revisión de extensos protocolos, y que las solicitudes realizadas por el patrocinador sobre cambios en las condiciones iniciales del protocolo no tuvieran el mismo nivel de acuciosidad en la revisión por dichas instancias ${ }^{(22)}$. A pesar de ello, es ineludible la responsabilidad de los investigadores involucrados, varios de ellos vacunados, con experiencia en la conducción de ensayos clínicos y en el ejercicio docente sobre temas de integridad científica y ética en investigación. Dicha experiencia y credenciales no fueron suficientes para realizar un análisis crítico propio sobre la situación de la que aceptaron ser parte.

El desarrollar un ensayo clínico en contexto de pandemia es complejo en nuestro país. Se conjugan varios factores que muchas veces colisionan entre sí, como generar oportunamente evidencia de la eficacia de la vacuna, asegurar la validez científica del estudio, cumplir los principios éticos, mantener la integridad científica y preservar la confianza de la sociedad en la investigación. Todo esto nos plantea enormes desafíos como país, comunidad científica y ciudadanía. Por tanto, después de estos hechos, se deberá trabajar en la recuperación de la confianza pública hacia la investigación científica y particularmente la investigación clínica. Queda pendiente una amplia reflexión de los investigadores sobre la integridad científica en ensayos clínicos y la investigación biomédica en general, cuestiones que no han podido ser revisadas en esta editorial ${ }^{(23)}$. Es indudable que estos hechos tienen suficientes aristas para ser material de estudio de caso en cursos de ética en investigación en el país y en el extranjero.

\section{Conflictos de interés}

Los autores declaran no tener conflictos de interés con la publicación de este artículo. 


\section{REFERENCIAS BIBLIOGRÁFICAS}

1. Singh JA, Upshur REG. The granting of emergency use designation to COVID-19 candidate vaccines: implications for COVID-19 vaccine trials. Lancet Infect Dis. 2021;21(4):e103-9. doi: 10.1016/S14733099(20)30923-3.

2. Chauvin L. Peruvian COVID-19 vaccine scandal spreads. Lancet. 2021;397(10276):783. doi: 10.1016/S0140-6736(21)00508-0.

3. Taylor L. Outrage over vaccine. Trial scandal at Peruvian universities. Nature. 2021;592(7853):174-5. doi: 10.1038/d41586-021-00576-0.

4. Ministerio de Salud del Perú. Informe de la comisión sectorial investigadora de la aplicación de la vacuna candidata contra la covid-19. Lima: MINSA; 2021.

5. Bermudez-Tapia M. Políticas públicas, pandemia y corrupción: el caso "vacunagate" en Perú. Rev Direitos Sociais Polit Publicas. 2021;9(1):984-1008.

6. Asundi A, Bhadelia N. Making emergency use of experimental vaccine safer. AMA J Ethics. 2020;22(1):e43-49. doi: 10.1001/amajethics.2020.43.

7. Jamrozick E, Selgelid MJ. COVID-19 human challenge studies: ethical issues. Lancet Infect Dis. 2020;20(8):e198-203. doi: 10.1016/S14733099(20)30438-2.

8. Fernandez Lynch H, Lundin D, Magher EA. Ethical inclusion of health care workers in covid-19 research. Ethics Human Res. 2021;43(2):1927. doi: 10.1002/eahr.500082.

9. Lhomme E, Modet C, Augier A, Faye S, Dabakuyo-Yonli TS, Levy-Marchal C, et al. Enrolling study personnel in Ebola vaccine trials: from guidelines to practice in a non-epidemic context. Trials. 2019;20:422. doi: 10.1186/s13063-019-3487-0.

10. Asociación Médica Mundial. Declaración de Helsinki - Principios éticos para la investigación en seres humanos [Internet]. Fortaleza, Brasil: AMM; 2017 [citado el 12 de junio de 2021]. Disponible en: https://www. wma.net/es/policies-post/declaracion-de-helsinki-de-la-amm-principios-eticos-para-las-investigaciones-medicas-en-seres-humanos/

11. Instituto Nacional de Salud. Reglamento de ensayos clínicos en el Perú. Lima: INS; 2006.

12. Borysowski J, Ehni HH, Gorski A. Ethics review in compassionate use. BMC Med. 2017;15:136. doi: 10.1186/s12916-017-0910-9.
13. Borysowski J, Gorski A. Compassionate use of unauthorized drugs: legal regulations and ethical challenges. Eur J Internal Med. 2019;65:12 16. doi: 10.1016/j.ejim.2019.04.008.

14. Instituto Nacional de Salud. Reglamento de ensayos clínicos en el Perú. Lima: INS; 2018.

15. Sharma A, Ahmed S, Kaur J, Chawla R, Rejeeth C. Exploring status of emergency drugs and vaccine development in covid-19 pandemic: an update. VirusDis. 2021;1-13. doi: 10.1007/s13337-021-00684-5.

16. Rizk JG, Forthal DN, Kalantar-Zadeh K, Mehra MR, Lavie CJ, Rizk Y, et al. Expanded access programs, compassionate drug use, and emergency use authorizations during the COVID-19 pandemic. Drug Discovery Today. 2021;26(2):593-603. doi: 10.1016/i.drudis.2020.11.025

17. John TJ, Dharmapalan D. Covid-19 vaccine trials and ethics: protection delayed is protection denied. Indian J Med Ethics. 2020;6(1):17-20.

18. Al Jazeera. UAE prime minister receives coronavirus vaccine shot [Internet]. Doha, Qatar: Al Jazeera; 2021 [citado el 12 de junio de 2021]. https://www.aljazeera.com/news/2020/11/3/uae-pm-and-dubai-ruler-receives-coronavirus-vaccine

19. Cabral E, Chavez Amaya C, Jara M, Velázquez K, Ruiz I. Laboratorios enviaron dosis adicionales y ofrecieron vacunación irregular en ensayos clínicos de América Latina [Internet]. Lima: Ojos Público; 2021 [citado el 12 de junio de 2021]. Disponible en: https://ojo-publico.com/2814/ dosis-extras-y-vacunacion-irregular-en-america-latina

20. Fernandez Lynch H, Dickert NW, Zettler PJ, Joffe S, Largent EA. Regulatory flexibility for covid-19 research. J Law Biosciences. 2020;7(1):110. doi:10.1093/jlb/lsaa057.

21. Bolislis WR, de Lucia ML, Dolz F, Mo R, Nagaoka M, Rodriguez H, et al. Regulatory agilities in the time of covid-19: overview, trends, and opportunities. Clin Therapeutics. 2021;43(1):124-39. doi: 10.1016/j. clinthera.2020.11.015.

22. Cahuina Lope P, Carracedo S, Romani F. La regulación de los ensayos clínicos para la covid-19 en el Perú. Rev Peru Med Exp Salud Publica. 2021;38(1):171-7. doi: 10.17843/rpmesp.2021.381.6627.

23. Arevalo J, Villafuerte FC, Montes M, Ochoa TJ. Peruvian research striving for the highest standards. Lancet. 2021;397(10287):1805-6. doi: 10.1016/S0140-6736(21)00890-4. 